$\Rightarrow$ MILESTONE 6

\section{Fly out of the traps}

Perhaps fittingly for a machine that works by sending beams of charged particles on undulating paths, the journey of the quadrupole mass filter from conception to laboratory bench was not particularly straightforward. Wolfgang Paul, at the University of Bonn, first published the concept behind the mass filter-or spectrometer-in 1953. Today, he is regarded as the technology's father. Less known, however, is that a researcher at the University of California (later Lawrence) Radiation Laboratory, Richard Post, came up with a similar idea around the same time. But he never published his work: his ideas made it only into his personal notebooks and a Lawrence Radiation Laboratory report.

Paul is known also for his development of quadrupole ion traps, for which he shared the Nobel Prize in Physics in 1989. Quadrupole ion traps and mass filters both use electric quadrupole fields to manipulate ionized atoms or charged particles. In ion traps, the ions are confined to a small region in which they can be laser cooled and used for spectroscopy, ultracold chemistry or quantum information processing, whereas quadrupole mass filters guide the ions to a detector. The filters consist of four metal rods that have both direct-current and oscillating radiofrequency voltages applied in a constant ratio between the opposing pairs. The exact nature of the trajectories of the ions depends on their mass-to-charge ratios. This ratio can be determined relatively easily, as different electric-field strengths and oscillating frequencies are required to transmit different species of ions through the electrode structure and onto the detector.

By the early 1960s, several companies were producing quadrupole mass filters, but widespread adoption of the technology was surprisingly slow. Compared with early magnetic sector analyzers, the mass filters were smaller, cheaper, tolerant of more extreme conditions and generally easier to automate-so why such resistance to them? Magnetic-based devices were trusted, and researchers knew both how they worked and how to use them. The quadrupole mass filters

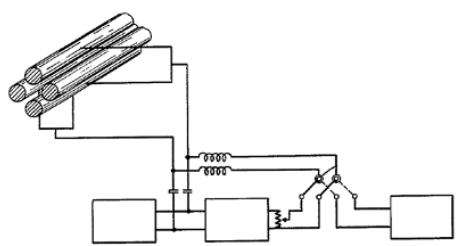

Diagram of Wolfgang Paul's patent for a quadrupole mass filter. Image adapted from US patent 2,939,952 A (1953). therefore needed to do something spectacular, beyond what was possible with conventional techniques. One person who saw such an opportunity was Robert Finnigan.

In 1967, Finnigan co-founded Finnigan Instruments, which aimed to combine these mass filters with gas chromatography

(Milestone 8) to achieve a single, computerized system that could separate and identify the constituents of a mixture. Importantly, quadrupole mass filters were able to generate mass spectra at unparalleled sampling speeds. This gave chemists, biochemists and countless others a simple system that could analyze samples within hours.

In the late 1970s, as quadrupole technologies became more widely adopted, Jim Morrison discovered an innovative use for them. With a line of three quadrupoles, a specific ion can be isolated in the first filter, broken into fragments in the second and then analyzed and detected in the third, providing a method for probing chemical structure or selecting and monitoring specific chemical reactions (Milestone 17). Morrison used light to break up ions, but this was not a practical approach for analytical studies.

Along with his student Richard Yost, Christie Enke showed that energetic gas-phase collisions in the second quadrupole could be used to break apart the ions, without the need for light. This collision-induced fragmentation was achieved by adding an inert gas, such as argon, to increase the pressure. Similar to what happens in particle accelerators, energetic ions, when accelerated by electric fields applied between the first and second quadrupoles, collide with these gas molecules and separate into smaller pieces.

Enke, Yost and Morrison realized that this triple-stage system could be extended to the analysis of more complex organic ions (see

Milestone 7), as well as to ions formed from proteins (see Milestone 20). And although the initial adoption of quadrupole mass filters was not as fast as one might expect for such a revolutionary technology, triple-stage quadrupole mass spectrometers rose quickly to popularity and are firmly established as invaluable tools for a range of disciplines in laboratories around the world.

Luke Fleet, Associate Editor, Nature Physics

ORIGINAL RESEARCH PAPERS Paul, W. \& Steinwedel, H. Ein neues Massenspektrometer ohne Magnetfeld. Zeitschrift für Naturforschung A. 8, 448-450 (1953) | Paul, W. Apparatus for separating charged particles of different specific charges. US patent 2,939,952 A (1953) | Yost, R.A., Enke, C.G., McGilvery, D.C., Smith, D. \& Morrison, J.D. High-efficiency collisioninduced dissociation in an RF-only quadrupole. Int. J. Mass Spectrom. Ion Phys. 30, 127-136 (1979) FURTHER READING Finnigan, R.E. Quadrupole mass spectrometers: from development to commercialisation. Anal. Chem. 66, 969A-975A (1994) 